\title{
Spinal intradural metastasis from scapular Ewing sarcoma
}

\author{
Dissanayake Mudiyanselage Priyantha Udaya Kumara Ralapanawa ${ }^{1 *}$, \\ Kushalee Poornima Jayawickreme ${ }^{1}$, Ekanayake Mudiyanselage Madhushanka Ekanayake ${ }^{1}$ and \\ Kulatunga Wijekoon Mudiyanselage Pramitha Prabhashini Kumarihamy²
}

\begin{abstract}
Background: Ewing sarcoma is a primary bone neoplasm, which is a high grade aggressive small round blue cell tumour, and is currently recognized as a part of the Ewing family of tumours. It is the most lethal bone tumor, and is a rare malignant bone tumor accounting for $10 \%$ of all primary bone tumors, and $6 \%$ of malignant bone tumors. It has an average annual incidence of 3 per 1 million, found almost exclusively in Caucasians. It commonly occurs in long bones and pelvis but rarely involves the scapula. $85 \%$ of cases have metastasis within 2 years of diagnosis, rarely involving the meninges.
\end{abstract}

Case presentation: We report a case of a 25 year old Sinhalese Sri Lankan female, presenting with a 1 day history of bilateral lower limb weakness and urinary incontinence. She had a sensory level with flaccid paralysis of lower limbs and a painless bony lump in the left dorsal scapula. Investigations showed scapular primary Ewing sarcoma giving rise to spinal intradural metastasis. For the best of our knowledge this is the first reported case of a scapular Ewing sarcoma with spinal intradural metastasis presenting with lower limb paralysis.

Conclusion: Intradural spinal metastasis of Ewing sarcoma presenting with lower limb weakness, without a history of pain, though rarely, can be the first presentation, and can rapidly progress to brainstem involvement and death.

Keywords: Ewing sarcoma, Flaccid paralysis, Scapular bone neoplasm, Spinal metastasis, Sri Lanka

\section{Background}

Ewing sarcoma (ES) is a primary bone neoplasm which is a high grade aggressive small round blue cell tumour, and is currently recognized as a part of the Ewing family of tumours. It was first described by famed pathologist James Ewing in 1921 [1, 2]. It commonly demonstrates reproducible staining of $\mathrm{CD} 99$ and translocations of the EWS gene. This neoplasm is usually associated with near certain metastasis at the time of diagnosis with a high rate of mortality. Occurrence of ES is frequently seen in the second decade of life in the diaphysis of long bones and pelvis; and has an average annual incidence of about 3 per 1 million. It has a slight predominance in males which accounts for $61 \%$ of the cases diagnosed, and is

\footnotetext{
*Correspondence: udayapralapanawa@yahoo.com

${ }^{1}$ Department of Medicine, University of Peradeniya, Peradeniya, Sri Lanka Full list of author information is available at the end of the article
}

found almost exclusively in Caucasians who represented $92 \%$ of the cases [2].

\section{Case presentation}

A 25 year old Sinhalese Sri Lankan female presented with a 1 day history of bilateral lower limb weakness, and numbness with urinary incontinence. She had no back pain and no history of constitutional symptoms such as fever, loss of appetite, or recent subjective weight loss.

On examination she had atonic lower limbs, with absent muscle power, and absent bilateral lower limb reflexes below knee level, with sensory impairment up to T6 level. She had no spinal deformities or tenderness, and no papilloedema. Upper limb examination was unremarkable except for a hard non tender bony mass on the left scapular region. She had a blood pressure of $140 / 80 \mathrm{mmHg}$, pulse rate of 78 beats per minute and had no respiratory compromise. 
She was investigated with a suspicion of metastatic disease and X-ray of the left shoulder showed a soft tissue and bony mass on the dorsal aspect of the left scapula with multiple lytic lesions suggestive of a primary bone neoplasm (Figure 1), but chest radiograph, ultrasound scan of the neck, and Computed tomography (CT) of abdomen were normal. Magnetic resonance imaging (MRI) of the spine showed an intradural extramedullary mass with an extra spinal component at C7-T2 level causing severe cord compression (Figure 2), hence intravenous dexamethasone regimen was started.

Ultrasound guided core needle biopsy from the left scapular mass showed malignant small round blue cell tumour suggestive of Ewing sarcoma. Blood investigations showed Heamoglobin of $9.2 \mathrm{~g} / \mathrm{dl}$, white blood cell count of $12 \times 10^{3} / \mu \mathrm{l}$, Erythrocyte sedimentation rate (ESR) of $150 \mathrm{~mm} / \mathrm{h}, \mathrm{C}$-reactive protein level of $96 \mathrm{mg} / \mathrm{l}$, normal liver enzyme levels and liver functions tests, and serum alkaline phosphate level of $173 \mu / 1$. Her blood picture showed increased rouleaux formation with anaemia of chronic disease.

She had no improvement of symptoms following treatment with dexamethasone. Before implementing on oncological management, 3 days after onset of symptoms, she developed sudden onset progressive ascending

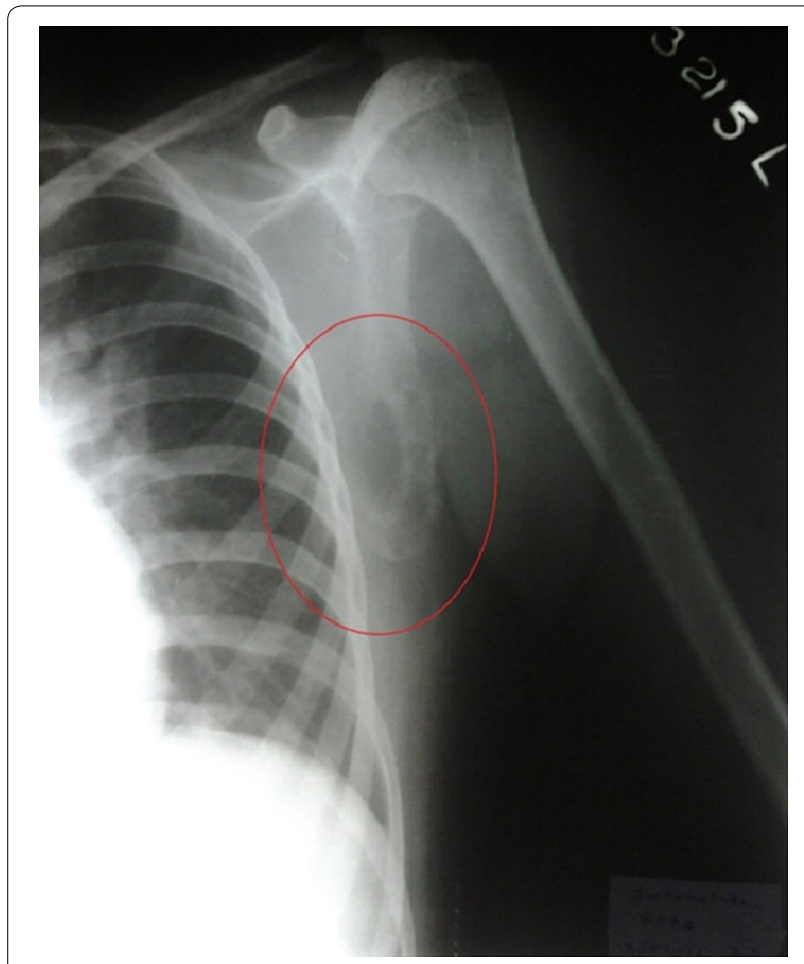

Figure 1 Radiograph of the left scapula. Radiograph of the left scapula showing a bony mass with multiple lytic lesions in the lateral and dorsal aspects.

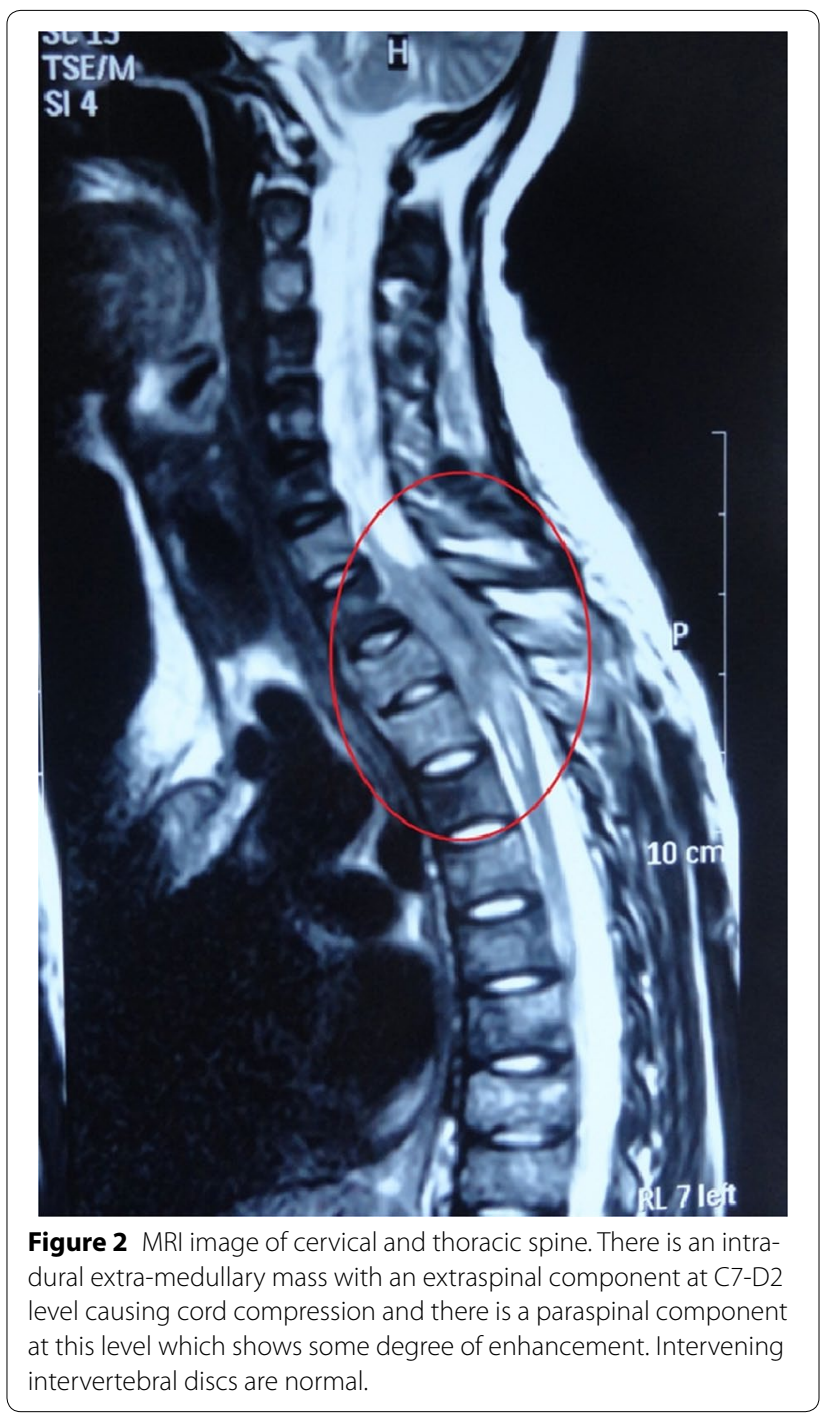

neurological impairment with upper limb and bulbar involvement, and unfortunately resulted with respiratory failure and death.

\section{Discussion}

$\mathrm{ES}$, which is the most lethal bone tumor, is a rare malignant bone tumor accounting for $10 \%$ of all primary bone tumors, and $6 \%$ of malignant bone tumors [3]. The commonest symptom of ES is a dull to severe pain which becomes persistent from about 1 month prior to diagnosis, and is seen in approximately $70 \%$ cases, unlike in this patient who had a painless mass [3]. Other features are fever, anemia, leukocytosis and increased ESR. ES is thought to be neuroectodermal in origin. More than $80 \%$ of ES occur during the first two decades of life, wherein Krieun has described the distribution of ES varying with age mirroring the distribution of marrow [4]. 
ES more commonly occurs in tubular bone than in flat bone, and the commonest affected site is the pelvis (25\%), followed in order of frequency by ribs, femurs, spine, tibias, fibulas, scapulas and other bones [4]. The primary site of tumor in this case was the scapula. However, malignant tumor of the scapula is rare, which may comprise of chondro-sarcoma, synovial sarcoma, ES, and metastasis [5]. This case presented with spinal intradural metastasis, whereas primary malignant sarcoma of the spine is rare accounting for $3.5-14.9 \%$ of primary bone sarcomas [6]. Metastasis occurs in up to $85 \%$ of patients within 2 years of diagnosis. Metastasis occurs to lungs (85\%), bones (69\%), pleura (46\%), lymph nodes $(46 \%)$, dura and/or meninges (27\%) and central nervous system (12\%) respectively [7].

Central nervous system involvement which is seen in $10-35 \%$ of patients occurs by either spinal cord compression or meningeal dissemination [8]. Most previous reports of central nervous system metastasis of ES showed involvement of the bony calvarium or brain paranchyma. Most such lesions were found to be located in an intracranial or extradural site, making epidural and intradural metastasis even rarer [9]. The commonest malignancies involving the spinal epidural space are metastatic lymphomas, nerve sheath tumors, meningiomas, hemangiomas and metastasis from systemic malignancies. Primary extra-skeletal ES occurring in a paravertebral location with a predilection to infiltrate through neural exit foramina have been rarely reported [10].

Microscopic characteristics typical of ES include, Periodic acid-Schiff positive, diastase-digestible cytoplasmic material, clear cytoplasm with indistinct cellular borders, and the uniformity of small oval blue nuclei [11]. A histology of small round cell bone tumor can be further confirmed as ES by immunohistochemical and cytogenetic analysis, which shows consistent chromosomal anomaly, the reciprocal translocation $t(11 ; 22)(\mathrm{q} 24 ; \mathrm{q} 12)$ [12]. ES is classically described radiologically as a central, diaphyseal, lytic or rarely sclerotic tumor, with "onion skin" type periosteal reaction affecting a long bone associated with soft tissue mass [13]. MRI, which is superior to CT, is frequently associated with a prominent soft tissue mass that contains areas of necrosis or hemorrhage [3].

The successful treatment of patients with ES requires systemic chemotherapy in conjunction with either surgery or radiation therapy or both modalities for local tumor control. Prognosis depends on the size and location of the tumor, presence or absence of tumor metastasis, tumor response to therapy, age, and disease relapse, out of which the presence of distant metastasis having the worst prognosis as seen in this patient. The current long term survival of ES is found to be $60-70 \%$. However, patients with metastasis only have a long term survival of $20 \%$, despite aggressive treatment. Histological grades have no prognostic significance, but the presence of fever, anaemia, leukocytosis, elevated ESR, and lactate dehydrogenase have been reported to indicate more extensive disease and a worse prognosis [13].

\section{Conclusion}

Intradural spinal metastasis of ES presenting with lower limb weakness, without a history of pain, though rarely, can be the first presentation, and can rapidly progress to brainstem involvement and death.

\section{Consent}

Written informed consent was obtained from the deceased patient's mother for publication of this case report and accompanying images. A copy of the written consent is available for review by the Editor-in-chief of this journal.

\section{Abbreviations}

ES: Ewing sarcoma; CT: computed tomography; MRI: magnetic resonance imaging; ESR: erythrocyte sedimentation rate.

\section{Authors' contributions}

Analysis and interpretation of patient data and literature review were done by DMPUKR, KPJ, EMME and KWMPPK. DMPUKR guided the other authors in reporting this case and corrected the final manuscript. All authors were involved in the management of the patient. All authors read and approved the final manuscript.

\section{Author details}

${ }^{1}$ Department of Medicine, University of Peradeniya, Peradeniya, Sri Lanka.

${ }^{2}$ University Medical Unit, Teaching Hospital Peradeniya, Peradeniya, Sri Lanka.

\section{Acknowledgements}

We express our gratitude to the deceased patient's mother, who kindly gave consent for this case to be presented in this paper.

\section{Compliance with ethical guidelines}

Competing interests

The authors declare that they have no competing interests.

Received: 14 May 2015 Accepted: 30 June 2015

Published online: 08 July 2015

\section{References}

1. Ewing J (1921) Diffuse endothelioma of bone. Proc NY Pathol Soc 21:17-24

2. Moore DD, Haydon RC (2014) Ewing's sarcoma of bone. Cancer Treat Res 162:93-115

3. Kara G (2004) Spinal cord Ewing's sarcoma metastasis: presentation of one case. Ann Nucl Med 18(7):623-626

4. Krieun ME (1985) Red-yellow marrow conversion: its effects on the location of some solitary bone lesions. Skelet Radiol 14:10-19

5. Hoornenborg D, Veltman ES, Oldenburger F, Bramer JAM, Schaap GR (2013) A patient with scapular Ewing sarcoma; 5-year follow-up after extracorporeal irradiation and re-implantation of the scapula, a case report. J Bone Oncol 2:30-32

6. Dini LI, Mendonça R, Gallo P (2006) Primary Ewing's sarcoma of the spine. ArqNeuropsiquiatr 64(3-A):654-659 
7. Hattori T, Yamakawa H, Nakayama N, Kuroda T, Andoh T, Sakai N et al (1999) Skull metastasis of Ewing's sarcoma: three case reports. Neurol Med Chir (Tokyo) 39:946-949

8. Mehta Y (1974) Hendrickson FR.CNS involvement in Ewing's sarcoma. Cancer 33:859-862

9. D'souza MM, Sharma R, Jaimini A, Tripathi M, Singh D, Mondal A (2012) Spinal epidural metastasis from Ewing's sarcoma on PET/CT: a case report. Rev Esp Med Nucl Imagen Mol 31(3):151-154

10. Ali S, Mackenzie K, Reid R, O’Neill G, Ganly I (2008) Cervical extraskeletal Ewing's sarcoma: case report demonstrating radiological features and management. J Laryngol Otol 122:998-1001
11. Navas-Palacinos JJ, Aparicio-Duque R, Valdes MD (1984) On the histogenesis of Ewing's sarcoma. An ultrastructural, immunohistochemical, and cytochemical study. Cancer 53:1882-1901

12. Turc-Carel C, Philip I, Berger MP, Philip T, Lenoir GM (1984) Chromosome study of Ewing's sarcoma (ES) cell lines. Consistency of a reciprocal translocation t(11;22)(q24;q12). Cancer Genet Cytogenet 12:1-19

13. Halwai MA, Mir BA, Wani MM, Bashir A, Hussain A (2009) Ewing's sarcoma of the ilium mimicking inflammatory arthritis of the hip: a case report. Cases Journal 2:6487
Submit your next manuscript to BioMed Central and take full advantage of:

- Convenient online submission

- Thorough peer review

- No space constraints or color figure charges

- Immediate publication on acceptance

- Inclusion in PubMed, CAS, Scopus and Google Scholar

- Research which is freely available for redistribution

Submit your manuscript at www.biomedcentral.com/submit 